racist attacks, reducing institutional racism, improving schooling, supporting the family, decreasing the number of Black children in care and offering people skills to deal appropriately with discrimination, could have such a positive impact on society that it would be a reasonable initiative for psychiatrists to support, even if there was only a modest direct decrease in the rates of mental illness in ethnic minority groups. However, we are aware that there is a danger that protracted, scientific attention given to empirical questions might overshadow consideration of the more important error which is the implication that socio-moral tenets can be appropriately derived from science. Concepts such as liberty, justice and freedom from discrimination are neither determined nor justified by scientific results but flow from constitutional and moral principles. Science has a role in social policy but, in this regard, it is less in defining rights and more in developing methods for achieving rights. Cost-benefit analysis of public health measures based on rights is a moral discourse. Given the difficulty in raising research funds for research into racism, waiting for the evidence, or opposing such initiatives because of the lack of 'evidence', is not a zero-sum game. We have no initiative to decrease the rates of mental illness in ethnic minority groups in the UK. Is it not time that we did?

Bhui, K. (ed.) (2002) Racism and Mental Health Prejudice and Suffering. London: Jessica Kingsley Publishers.

Boydell, J., van Os., J., McKenzie, K., et al (2001) Incidence of schizophrenia in ethnic minorities in London: ecological study into interaction with environment. BMJ, 323, 1336-1338.

Chakraborty, A. \& McKenzie, K. (2002) Does racial discrimination cause mental illness? British Journal of Psychiatry, 180, 475-477.

McKenzie, K. (1999) Rates of anxiety and depression in African-Caribbeans may not reflect reality (letter). BMJ, 319, 1007.

Sharpley, M. S., Hutchinson, G., Murray, R. M., et al (200I) Understanding the excess of psychosis among the African-Caribbean population in England: Review of current hypotheses. British Journal of Psychiatry, 178 (suppl. 40), s60-s68.

K. McKenzie Department of Psychiatry and Behavioural Sciences, Royal Free and University College Medical Schools, Royal Free Campus, Rowland Hill Street, London NW3 2PF, UK

A. Chakraborty St Ann's Hospital, London, UK

\section{Neuroticism and depression}

Farmer et al (2002) draw conclusions that we believe are not supported by the results of their study. The study compared probands with depression and their siblings, with healthy probands with no history of depression and their siblings. Between two-thirds and three-quarters of the participants were women. The groups did not differ in age that varied from 36.2 to 39.1 years.

The absence of a difference in mean scores for neuroticism for the neverdepressed siblings of both the healthy probands and those with depression was interpreted to suggest that this scale does not measure a genetically influenced trait for depression. This finding, however, can be interpreted otherwise. As the siblings of the probands with depression were in their mid-thirties and had not, as yet, experienced a depression, it is reasonable to assume that they have passed the age of risk for a first episode (Burke et al, 1990) and therefore may not have inherited a vulnerability for the disorder. The finding that the siblings of the probands with depression obtained scores for the trait of neuroticism similar to those obtained by siblings of the healthy probands, who presumably are not genetically vulnerable to depression, could be interpreted to suggest that neuroticism is necessary for depression. As long as there is no way to determine whether an individual carries the genes associated with depression, a cross-sectional study of adults cannot ascertain whether neuroticism reflects a part of the genetic vulnerability for depression. A prospective, longitudinal investigation in which the trait, or an ageappropriate proxy for the trait, is measured before the onset of symptoms could untangle the relationship between neuroticism and depression. Studies comparing monozygotic and dizygotic twins could also address the issue, as did Kendler and colleagues (Kendler et al, 1993) who reported that the genetic liability for major depression largely overlapped with that for neuroticism. The finding that neuroticism scores are positively correlated with symptoms of depression and with severe life events does not address the aetiological question.

It is important to determine whether the trait of neuroticism reflects a part of the genetic vulnerability for depression. It is also important to identify the factors that exacerbate the inherited vulnerability and lead to depression, in order to design prevention programmes for children at risk for depressive disorders. Among parents with a major affective disorder, neuroticism may have more influence on the development of their offspring than does the severity of their disorder. In a prospective study of the children of parents with bipolar disorder, we have found that neuroticism is associated with high levels of negative life events, low levels of psychosocial functioning and with poor parenting, which in turn are associated with the children's level of psychosocial functioning and symptoms (Hodgins et al, 2002; further details available from the author upon request). By contrast, none of the indices of the severity of the parents' disorder is associated with psychosocial functioning or symptoms of the offspring. These findings suggest that neuroticism, rather than the disorder, influences parental behaviours that impact on the mental health of the offspring.

In light of the above considerations, we believe that Farmer et al's 'Clinical implication' that 'Neuroticism reflects subclinical or residual symptoms of depression' is misleading. Among adult patients, symptoms of depression do appear to be associated with scores for neuroticism, as has been reported previously (Sauer et al, 1997). Whether or not the trait of neuroticism also represents a risk factor for depression, however, is not known. The cross-sectional study reported by Farmer et al (2002) does not address this important question. Available data suggest that the trait of neuroticism may play a critical role in the development of depressive disorders, conferring an inherited vulnerability and leading to parental behaviours associated with impaired functioning among the offspring.

Burke, K. C., Burke, J. D. Jr., Regier, D. A., et al (1990) Age at onset of selected mental disorders in five community populations. Archives of General Psychiatry, 47, $511-518$.

Farmer, A., Redman, K., Harris T., et al (2002) Neuroticism, extraversion, life events and depression The Cardiff Depression Study. British Journal of Psychiatry, 181, 118-122

Hodgins, S., Faucher, B., Zarac, A., et al (2002) Children of parents with bipolar disorder: A population at high risk for major affective disorders. Child and Adolescent Psychiatric Clinics of North America, II, 533-553.

Kendler, K. S., Neale, M. C., Kessler, R. C., et al (1993) A longitudinal twin study of personality and major depression in women. Archives of General Psychiatry, 50, 853-862.

Sauer, H., Richter, P., Czernik, A., et al (1997) Personality differences between patients with major depression and bipolar disorder: the impact of minor 
symptoms on self-ratings of personality. Journal of Affective Disorders, 42, 169-177.

S. Hodgins, M. Ellenbogen Department of Psychology, Université de Montréal, C.P: 6128, Succ. Centre Ville, Montréal, Québec H3C 3J7, Canada

Farmer et al (2002) examined whether selfreports of neuroticism and extraversion represent enduring vulnerabilities to depression. While their findings make a valuable contribution, their conclusions regarding neuroticism warrant additional consideration. They found variables reflecting past and current depression were significant predictors of neuroticism and interpreted this as inconsistent with their hypothesis that neuroticism would 'exhibit trait-like qualities' and would not be 'substantially influenced by alteration in mood-state' (p. 118). Farmer et al's findings did not directly address this hypothesis because they did not use a longitudinal design needed to observe fluctuations in mood-state. None the less, several longitudinal studies indicate that neuroticism is affective-state dependent. For example, Hirschfeld et al (1983) found that patients in remission from depression reported lower levels of neuroticism than they originally reported when depressed. Findings of this nature have been used to argue that neuroticism and related traits are only contaminants of depression. More recently, investigators (e.g. Santor et al, 1997) studying samples of patients have noted that absolute changes in depression-related traits are associated with changes in mood (i.e. affective-state dependent), but that there is also a consistency in the rank of patients with regard to their scores on these measures (i.e. relative stability). Findings such as these have been interpreted as indicating that depression-related personality traits have both state-like and trait-like properties.

Given the large association between neuroticism and depression, Farmer et al suggested that neuroticism may be largely 'a proxy measure for present or past depression' (p.121) and questioned whether neuroticism reflects a vulnerability for depression. Neuroticism refers to a tendency to experience negative affect, so this high degree of overlap is not surprising. More importantly, longitudinal studies (Hirschfeld et al, 1989; Krueger et al, 1996) have found that high premorbid neuroticism is positively associated with the development of depression.

In summary, Farmer et al's conclusion that neuroticism does not measure a vulnerability to depression and primarily reflects symptoms of depression is not warranted. Self-reports of neuroticism prospectively predict depression. Longitudinal studies support Farmer et al's conclusion that neuroticism is strongly associated with a person's current affective state. However, such studies also suggest that neuroticism is likely to have trait-like properties in addition to the state-like properties noted by Farmer et al.

Farmer, A., Redman, K., Harris, T., et al (2002) Neuroticism, extraversion, life events and depression. The Cardiff Depression Study. British Journal of Psychiatry, 18I, I18-122

Hirschfeld, R. M. A., Klerman, G. L., Clayton, P. J., et al (1983) Assessing personality: effects of the depressive state on trait measurement. American Journal of Psychiatry, 140, 695-699.

_ , _ , Lavori, P., et al (1989) Premorbid personality assessments of first onset of major depression. Archives of General Psychiatry, 46, 345-350.

Krueger, R. F., Caspi, A., Moffitt, T. E., et al (1996) Personality traits are differentially linked to mental disorders: a multi-trait-multi-diagnosis study of an adolescent birth cohort. Journal of Abnormal Psychology, 105, 299-312.

Santor, D. C., Bagby, R. M. \& Joffe, R.T. (1997) Evaluating stability and change in personality and depression. Journal of Personality and Social Psychology, $\mathbf{7 3}$ 1354-1362.

L. McWilliams University of Manitoba, PZ-430 PsycHealth Centre, 77I Bannatyen Avenue, Winnipeg, Manitoba, Canada R3E 3N4

Authors' reply: We are pleased to respond to the comments of Drs Hodgins \& Ellenbogen and Dr McWilliams and are grateful for their interest in our work.

Hodgins and Ellenbogen suggest that an absence of a difference in mean scores for neuroticism (N) for never-depressed siblings of probands with depression and never-depressed siblings of healthy controls can be interpreted as showing that the siblings of probands with depression have not inherited the vulnerability for the disorders. However, this is missing the point. We hypothesised that scores represent a genetically influenced trait that underpins the risk of developing depression in the presence of precipitating factors such as adverse life events. If $\mathrm{N}$ were such a trait, then it would be expected that all first-degree relatives of probands with depression who share an average $50 \%$ of their genes with their relative with depression, would have higher mean scores than subjects without such a genetic relationship to a proband with depression, irrespective of affective status. Our failure to detect a difference for $\mathrm{N}$ scores is not due to lack of power since we have shown differences between the relatives of probands with depression and controls for other personality measures (Farmer et al, 2003) such as the Harm Avoidance Scale of the Temperament and Character Inventory (Cloninger et al, 1993).

Both Hodgins \& Ellenbogen and McWilliams rightly point out that longitudinal studies may help to disentangle the relationship between $\mathrm{N}$ and depression. However, even longitudinal studies can fail to answer the issue of what came first, the chicken of neuroticism or the egg of depression. For example, it is now well recognised that depressive symptoms occur in children and adolescents as well as in adults. Consequently, in order to demonstrate that $\mathrm{N}$ scores represent an underlying vulnerability to depression and are not merely a proxy measure of depressive symptoms, it is necessary to show that elevated $\mathrm{N}$ scores occur in the absence of significant depressive symptoms at the first point of measurement. To our knowledge, no longitudinal study to date has shown this.

Our study is in keeping with a growing literature showing that $\mathrm{N}$ has considerable state-dependent as well as trait-like properties. Despite this, there remains a cherished belief that the measure does indeed represent a trait underlying the vulnerability to depression. We have not demonstrated such properties for the scale in our Cardiff study.

\section{Declaration of interest}

The Cardiff Depression Study was funded by a Project Grant from the Wellcome Trust.

Cloninger C. R., Svrakic, D. M. \& Przybeck, T. R. (1993) A psychobiological model of temperament and character. Archives of General psychiatry, 50, 975-989.

Farmer, A. E., McGuffin, P., Mahmood, A., et al (2003) A sib-pair study of the temperament and character inventory in major depression. Archives of General Psychiatry, in press.

A. Farmer, P. McGuffin Social, Genetic and Developmental Psychiatry Research Centre, Institute of Psychiatry, De Crespigny Park, Denmark Hill, London SE5 8AF, UK 